\title{
Avaliação da lordose lombar e sua relação com a dor lombopélvica em gestantes
}

\author{
Evaluation of lumbar lordosis and its relation to the lumbopelvic pain in pregnants \\ Beatriz da Silva Firmento', Ana Silvia Moccellin², Maria Alice Seghessi Albino', Patricia Driusso³
}

\begin{abstract}
RESUMOIO objetivo deste trabalho foi avaliar a magnitude da lordose lombar, sua influência na dor lombopélvica e a qualidade de vida em gestantes. Para tal, foi realizado um estudo com 20 mulheres não gestantes (C) e 13 gestantes ao longo dos trimestres gestacionais (G1, G2 e G3). Todas as mulheres foram submetidas à avaliação inicial para registro dos dados pessoais, hábitos de vida, antecedentes pessoais, uso de medicamentos, história ginecológica e obstétrica. Posteriormente, as voluntárias do grupo controle foram avaliadas uma vez e as gestantes foram avaliadas em três momentos distintos, no $1^{\circ}, 2^{\circ}$ e $3^{\circ}$ trimestres gestacionais. A avaliação do grau de lordose lombar foi realizada por meio de técnica fotogramétrica; a avaliação de locais de dor, o tipo de dor e sua intensidade foram feitas por meio do Questionário McGill de dor; e a avaliação da qualidade de vida foi feita pelo Questionário WHOQOL-bref. Neste trabalho, não foi possível observar padrão de alteração da curvatura lombar no decorrer da gestação. Também não foi observada relação entre a curvatura lombar e a dor lombopélvica relacionada à gestação.
\end{abstract}

Descritores I gravidez; curvaturas da coluna vertebral; dor lombar.

\begin{abstract}
IThe purpose of this study was to evaluate the magnitude of lumbar lordosis, its influence on lumbopelvic pain and quality of life in pregnant women. To this end, a study was done with 20 non-pregnant women (C) and 13 pregnant women during the trimesters of pregnancy (G1, G2 and G3). All women underwent initial assessment for registration of personal data, lifestyle, personal history, medications, gynecological and obstetric history. Later, the volunteers in the control group were evaluated once and pregnant women were evaluated at three different times, the first, second and third trimesters of pregnancy. The evaluation of the degree of lumbar lordosis was performed by a photogrammetric technique. The assessment of points/places of pain, the kind of pain and its intensity were made by McGill Pain Questionnaire, and the quality of life assessment was made by WHOQOL-bref. In this study, it was not possible to observe a pattern of change in lumbar curvature during pregnancy. There was also no relationship between lumbar curvature and lumbopelvic pain related to pregnancy.
\end{abstract}

Keywords I pregnancy; spinal curvatures; low back pain.

Estudo desenvolvido no Laboratório de Avaliação e Intervenção Fisioterapêutica sobre a Saúde da Mulher - Departamento de Fisioterapia da Universidade Federal de São Carlos (UFSCar) - São Carlos, SP, Brasil.

'Fisioterapeutas; Graduadas pela UFSCar - São Carlos (SP), Brasil.

2Fisioterapeuta; Mestre e Doutoranda em Fisioterapia pelo Programa de Pós-Graduação da UFSCar - São Carlos (SP), Brasil.

${ }^{3}$ Fisioterapeuta; Professora Doutora do Departamento de Fisioterapia da UFSCar - São Carlos (SP), Brasil. 


\section{INTRODUÇÃO}

Durante os nove meses de gravidez, o corpo feminino sofre uma série de alterações hormonais e anatômicas. Muitas destas alterações contribuem para a ocorrência de disfunções musculoesqueléticas, sendo a dor lombopélvica a de maior preocupação ${ }^{1}$. Estudos mostram que aproximadamente metade de todas as gestantes apresenta esta dor ${ }^{2,3}$.

Acredita-se que os principais motivos para a ocorrência da dor lombopélvica na gestação sejam as adaptações da coluna vertebral, que se devem, principalmente, à ação do hormônio relaxina e ao aumento considerável do peso do abdômen ${ }^{3-6}$.

O hormônio relaxina é considerado o principal responsável pela frouxidão ligamentar durante a gravidez $z^{1}$ o que permite que a sínfise púbica e a articulação sacro-ilíaca tornem-se mais flexíveis para a passagem do feto, levando à redução da estabilidade pélvica ${ }^{1,7}$.

Muitos autores ${ }^{1,8,9}$ acreditam que o peso adquirido na gestação acrescido da frouxidão ligamentar provocaria uma rotação anterior da pelve ${ }^{10}$. Para manter a projeção do centro de massa na área de sustentação na posição ereta, a região torácica se inclinaria posteriormente, acentuando a lordose lombar ${ }^{4}$, e isto poderia ocasionar a dor lombar por sobrecarga das estruturas da coluna vertebral ${ }^{11}$. Porém, estes mecanismos de compensação postural não estão totalmente elucidados, já que existem controvérsias entre os estudos que avaliam a postura das gestantes ${ }^{12-14}$.

A dor lombopélvica durante a gestação pode levar a inúmeras alterações na vida destas mulheres ${ }^{15}$ como limitação nas atividades da vida diária, qualidade de sono, disposição física, lazer e capacidade de traba1 ho ${ }^{16,17}$. Na literatura foi encontrado um estudo ${ }^{18}$ sobre a qualidade de vida de gestantes de baixo risco. Em relação às gestantes com dores nas costas, somente um estudo ${ }^{19}$ avaliou sua qualidade de vida. Além disso, não foi encontrada referência sobre a utilização do Questionário Word Health Organization Quality of
Life Instrument-bref (WHOQOL-bref), para avaliar a qualidade de vida em gestantes saudáveis ou com dor lombar. O WHOQOL-bref é um importante instrumento para avaliação da qualidade de vida que pode ser utilizado em diversos grupos e situações ${ }^{20}$.

Devido ao impacto que a dor lombopélvica pode causar na qualidade de vida das gestantes, o presente estudo teve por objetivo avaliar a magnitude da lordose lombar, sua influência no aparecimento da dor lombopélvica e a qualidade de vida durante os diferentes trimestres gestacionais.

\section{METODOLOGIA}

Tratou-se de um estudo descritivo transversal.

O estudo foi realizado de acordo com as normas regulamentadoras e aprovado pelo Comitê de Ética e Pesquisas em Seres Humanos da Universidade Federal de São Carlos (UFSCar), sob o Parecer no 280/2009.

Todas as voluntárias assinaram o Termo de Consentimento Livre e Esclarecido. O critério de inclusão determinou que todas as voluntárias apresentassem idade entre 18 e 40 anos, fossem saudáveis, não fumantes, não etilistas e nem apresentassem cirurgias na coluna vertebral, pelve, quadril ou joelho e nenhuma disfunção musculoesquelética ou neurológica. Além disso, as gestantes deveriam apresentar gestação de baixo risco e de feto único, sem intercorrências do ciclo gravídico. Vinte e quatro gestantes advindas de Unidades de Saúde da Família (USF) ou encaminhadas por médicos particulares do município de São Carlos (SP) interessaram-se por participar do estudo; 11 deixaram o estudo ou não completaram o protocolo de avaliação. Do total, 13 gestantes concluíram o estudo. Para estabelecimento de um controle basal foram analisadas 20 mulheres não gestantes, constituindo o grupo controle. A Tabela 1 apresenta as características antropométricas dos grupos controle e gestante.

Tabela 1. Características antropométricas das participantes dos Grupos

$\begin{array}{lcccc}\text { Características } & \begin{array}{c}\text { Controle } \\ \text { Média (DP) }\end{array} & \begin{array}{c}10 \text { trimestre } \\ \text { Média (DP) }\end{array} & \begin{array}{c}2^{\circ} \text { trimestre } \\ \text { Média (DP) }\end{array} & \begin{array}{c}3^{\circ} \text { trimestre } \\ \text { Média (DP) }\end{array} \\ \text { Semana gestacional } & - & 13,08 \pm 2,14 & 23,23 \pm 1,59 & 33,38 \pm 1,19 \\ \text { Idade (anos) } & 26,07 \pm 3,98 & 29,15 \pm 5,64 & 29,23 \pm 5,79 & 29,46 \pm 5,83 \\ \text { Altura (m) } & 1,63 \pm 0,07 & 1,64 \pm 0,09 & 1,64 \pm 0,09 & 1,64 \pm 0,09 \\ \text { Peso }(\mathrm{kg}) & 59,21 \pm 10,75 & 66,24 \pm 13,34 & 71,26 \pm 13,49 & 76,73 \pm 14,93 \\ \text { IMC }\left(\mathrm{kg} / \mathrm{m}^{2}\right) & 22,25 \pm 3,60 & 24,60 \pm 4,39 & 26,52 \pm 4,33 & 28,47 \pm 4,66\end{array}$




\section{Materiais e procedimentos}

Todas as mulheres foram submetidas à avaliação inicial, feita pelo próprio pesquisador, por meio de entrevista individual, utilizando um questionário para registro dos dados pessoais, hábitos de vida, antecedentes pessoais, uso de medicamentos, história ginecológica e obstétrica.

Posteriormente, as voluntárias do grupo controle (GC) foram avaliadas uma vez, para comparação com o primeiro trimestre gestacional das gestantes. As gestantes foram avaliadas entre a 10a $-14^{\mathrm{a}}(\mathrm{G} 1), 22^{\mathrm{a}}-24^{\mathrm{a}}(\mathrm{G} 2)$ e 32a-34a (G3) semanas gestacionais, de acordo com a data da última menstruação ${ }^{21} \mathrm{e} / \mathrm{ou}$ do primeiro ultrassom realizado durante a gestação ${ }^{22}$. O Índice de Massa Corporal das gestantes foi calculado com base na Tabela de Atalah ${ }^{23}$ adaptada.

Para a mensuração do grau de lordose lombar, foi fixado um marcador cutâneo sobre o processo espinhoso da décima segunda vértebra torácica (T12) e outro sobre o processo espinhoso da primeira vértebra sacral $(\mathrm{S} 1)^{24}$. Com a voluntária na posição ortostática, estas vértebras foram localizadas por palpação de acordo com Hoppenfeld ${ }^{25}$. Com uma máquina fotográfica digital (FinePix A345, 4.1 Mega Pixels, Fujifilm) foi realizado o registro fotográfico dessas marcações, com as voluntárias na posição lateral direita e braços cruzados na altura do tórax.

A mensuração do grau de lordose lombar foi realizada por meio de uma técnica fotogramétrica que tem mostrado algumas vantagens sobre outras utilizadas para o mesmo fim, já que é uma técnica não invasiva, de fácil aplicação e baixo custo, além da sua alta confiabilidade ${ }^{24}$.

Os marcadores utilizados nesse estudo, constituídos por uma base acrílica, no formato quadrado, tinham $3 \mathrm{~cm}$ de comprimento e de largura. No centro da base era fixada uma haste metálica, perpendicular, de $7 \mathrm{~cm}$ de comprimento, envolvido por um adesivo refletor. A distância entre os dois pés era de $20 \mathrm{~cm}$. A máquina estava fixada em um tripé com altura de $50 \mathrm{~cm}$ e com distância de $1,3 \mathrm{~m}$ da voluntária. A fotografia foi transferida para o computador e salva como um documento, no formato JPG.

Os registros fotográficos foram inseridos no software AUTOCAD-R14, no qual foi possível traçar as retas paralelas aos marcadores de T12 a S1 e definir o ângulo formado entre elas e que representou a medida do grau de lordose lombar.

O tipo de dor e sua intensidade foram avaliados pelo Questionário McGill de dor. O questionário McGill de dor é um dos melhores instrumentos para a avaliação das dimensões sensitiva-discriminativa, afetiva-motivacional e cognitiva-avaliativa da dor ${ }^{26}$. A versão brasileira apresenta 68 palavras e é composta por 4 partes: 1 - Localização da dor; 2 - Padrão temporal da dor; 3 - Descrição da dor; e 4 - Intensidade da dor presente $(\mathrm{IDP})^{27}$. A avaliação da qualidade de vida foi realizada pelo Questionário WHOQOL-bref, composto por 24 questões abrangendo os domínios: físico, psicológico, relações sociais e meio ambiente e duas questões gerais de qualidade de vida ${ }^{28,29}$.

\section{Análise estatística}

As informações tabuladas foram exportadas para o software Statistica, onde foi realizada a análise dos dados. Os dados foram analisados por meio de testes não paramétricos: a comparação entre os três trimestres (G1, G2 e G3) foi realizada pelo teste de Friedman e, nos casos significantes, utilizou-se o teste de Wilcoxon para discriminar a diferença. Para a comparação entre as gestantes do primeiro trimestre (G1) e não gestantes (C) utilizou-se o teste de Mann-Whitney. Foi adotado um nível de significância de 5\%.

Foi determinado o Índice de Mudança Confiável proposto por Jacobson e Truax ${ }^{30}$ como parte integrante do Método JT. Este índice é um indicador de erro da medida obtida na avaliação do participante, que é comparado a uma distribuição teórica de erros do instrumento. Para isso, os autores do Método JT desenvolveram uma fórmula específica, baseada na diferença entre pré-teste e pós-teste, dividida pelo erro padrão da diferença ${ }^{31}$, sendo que o erro padrão da diferença é representado pelo desvio padrão pré-teste, multiplicado pela raiz de 2 e pela raiz de 1 menos o índice de confiabilidade do instrumento, normalmente, o Coeficiente de Cronbach.

\section{RESULTADOS}

O Gráfico 1 representa os valores do grau de lordose lombar na vista lateral direita no primeiro e no último trimestre da gestação, além do Intervalo de Confiança para Mudança Confiável.

No Gráfico 2 são apresentados os números de queixas para cada região assinalada pelas gestantes na vista anterior e posterior do Questionário McGill de dor. A queixa mais frequente, durante os trimestres gestacionais, foi na coluna lombar. 


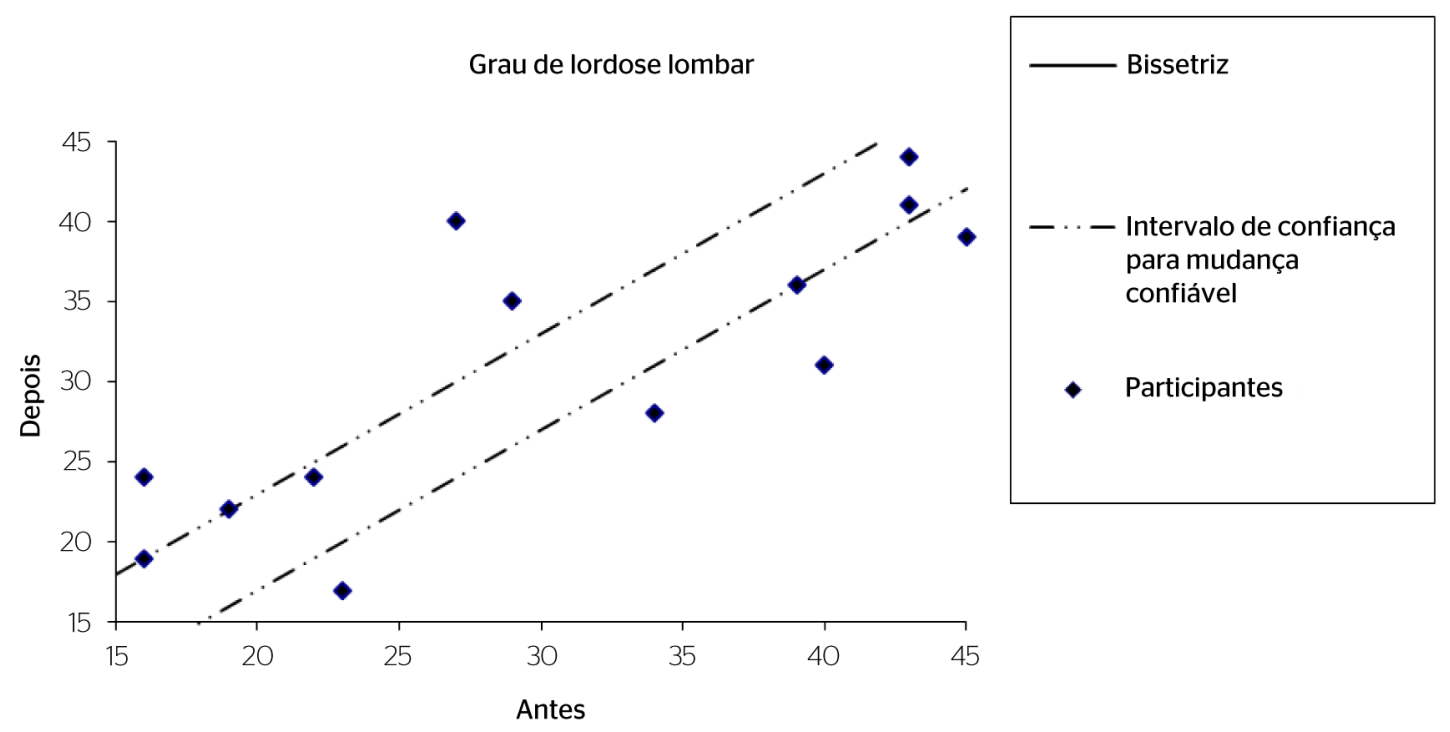

Gráfico 1. Representação gráfica da variável grau de lordose lombar ( ${ }^{\circ}$ ) da vista lateral direita para cada gestante no primeiro trimestre (Antes) e no terceiro trimestre (Depois) e intervalo de confiança para mudança confiável

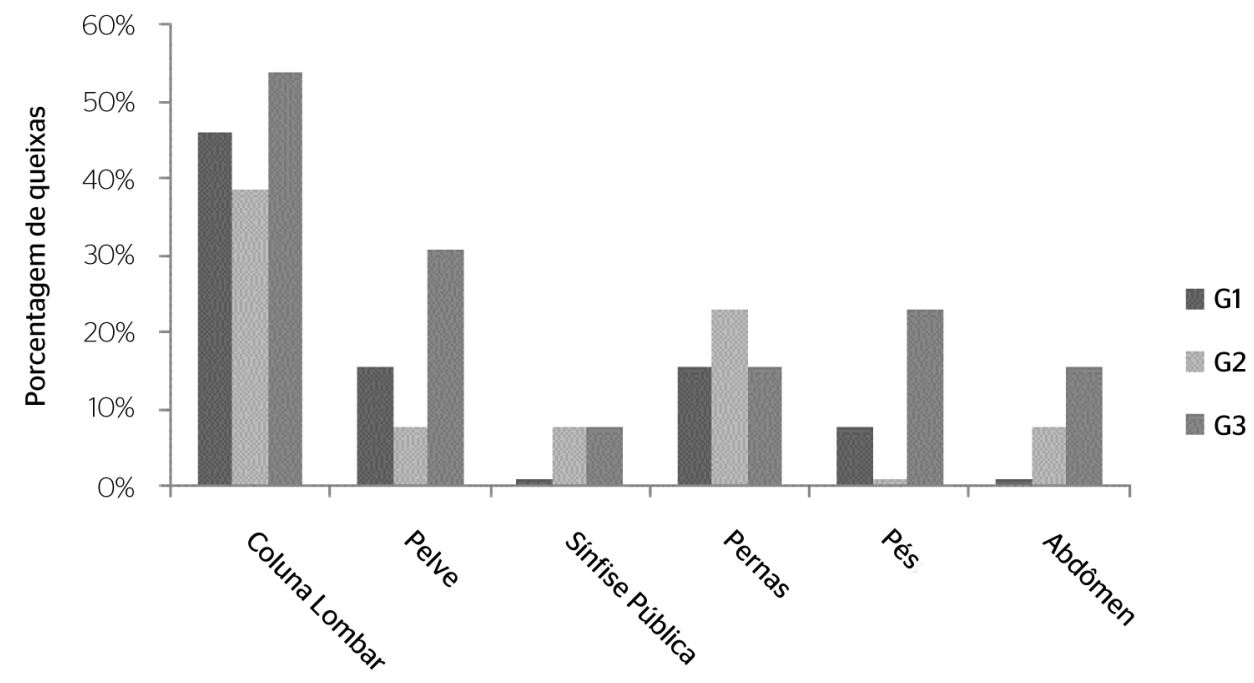

Gráfico 2. Porcentagem de queixas na coluna lombar, pelve, sínfise púbica, pernas, pés e abdômen no primeiro (G1), segundo (G2) e terceiro (G3) trimestres gestacionais

O Gráfico 3 representa os escores do Questionário McGill de dor no primeiro e no último trimestre da gestação, além do Intervalo de Confiança para Mudança Confiável.

No Gráfico 4 estão representados os escores dos quatro domínios do WHOQOL-bref: físico, psicológico, relações sociais e meio ambiente para o grupo controle, primeiro, segundo e terceiro trimestres. Houve diferença significativa apenas na comparação do grupo controle com o primeiro trimestre gestacional no escore do domínio físico ( $\mathrm{p}=0,016)$.

\section{DISCUSSÃO}

Este estudo teve por objetivo avaliar a magnitude da lordose lombar, sua influência no aparecimento da dor lombopélvica e a qualidade de vida durante os diferentes trimestres gestacionais.

Diferente do que muitos autores acreditam ${ }^{1,4,8,9}$, neste trabalho não foi possível observar um padrão de alteração da curvatura lombar, já que algumas mulheres apresentaram redução, outras aumento e outras, ainda, praticamente não sofreram alteração na lordose 


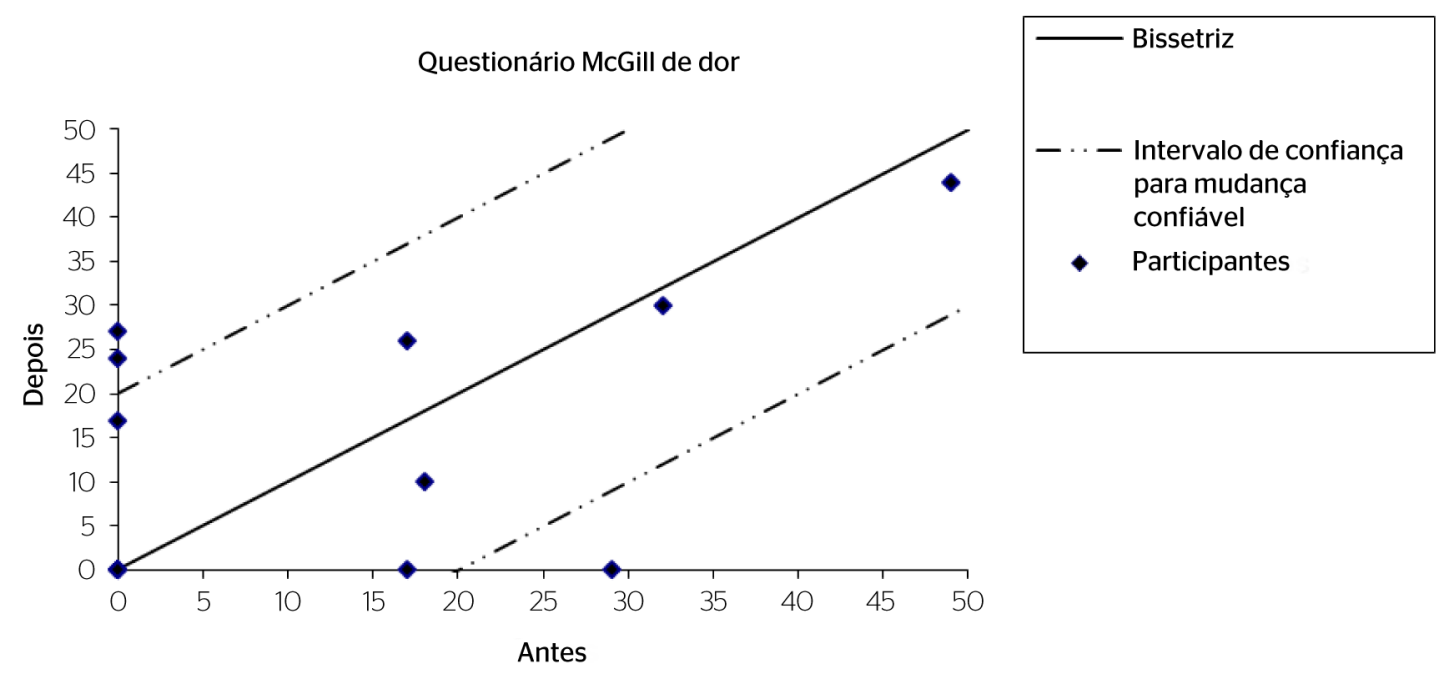

Gráfico 3. Representação gráfica da variável escore do Questionário McGill de dor para cada gestante no primeiro trimestre (Antes) e no terceiro trimestre (Depois) e intervalo de confiança para mudança confiável

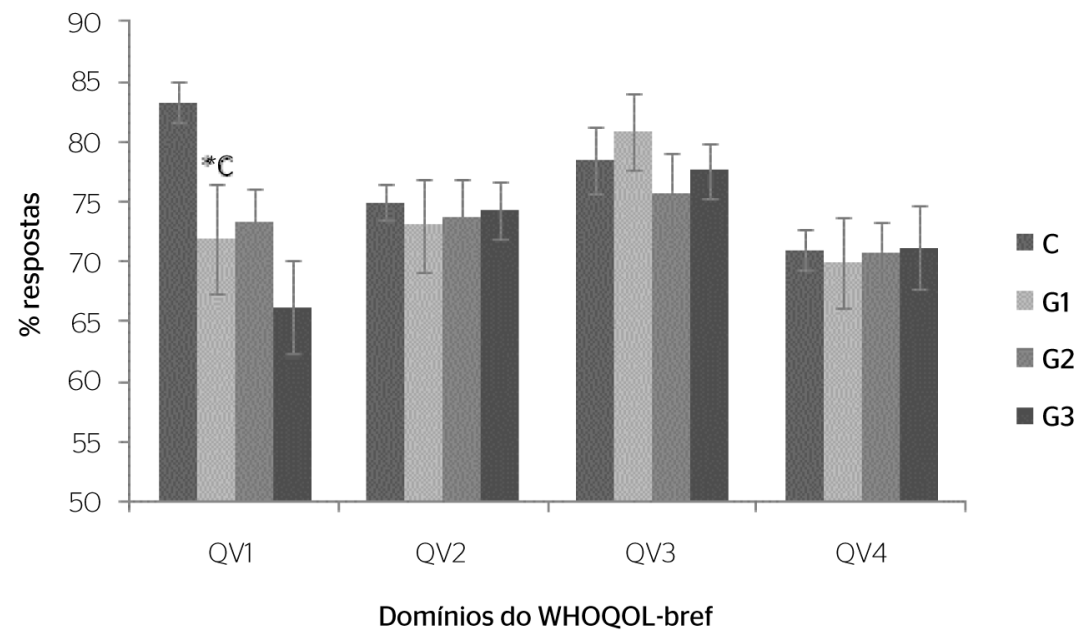

QV1: Domínio físico; QV2: Domínio psicológico; QV3: Relações sociais; QV4: Meio ambiente. *significativo Mann-Whithey $(p<0,05)$

Gráfico 4. Escore das respostas (\%) dos quatro domínios do WHOQOL-bref no grupo controle (C), primeiro (G1), segundo (G2) e terceiro (G3) trimestres gestacionais.

do primeiro para o terceiro trimestre. Esses diferentes comportamentos posturais entre as gestantes também foram relatados em outros estudos ${ }^{32,33}$.

Ao contrário do que foi encontrado neste trabalho, Benetti et al. ${ }^{4}$, Franklin \& Conner-Keer ${ }^{34}$ e Bullock-Saxton ${ }^{35}$ encontraram aumento significativo da curvatura lombar durante a gravidez. Porém, não há consenso na literatura sobre esta alteração, já que estudos ${ }^{12-14,32}$ utilizando outros métodos de avaliação não encontraram aumento significativo nos graus da lordose lombar.

É possível que ocorram outras alterações posturais não relacionadas ao aumento da lordose lombar em gestantes, para compensar o momento de flexão causado pelo ganho de volume abdominal, como hiperextensão da articulação do quadril ${ }^{13,22,36}$, da cervical ${ }^{13}$, ou ainda um deslocamento posterior de todo o corpo, em forma de pêndulo invertido com o centro de rotação localizado no tornozelo ${ }^{12}$.

Neste estudo, a relação entre alterações na curvatura lombar e a dor lombopélvica não ficou bem esclarecida, assim como no estudo de Bullock-Saxton ${ }^{35}$. Alguns estudos ${ }^{13,14}$ encontraram fraca correlação entre o aumento da lordose e a dor lombar. Já Dumas et al. encontraram correlação entre a dor e a curvatura lombar somente no primeiro trimestre ${ }^{37}$.

O aumento da lordose lombar é muito citado como uma das causas da dor lombopélvica relacionada à gestação, porém, muitos estudos ${ }^{33,34,38,39}$ não encontraram uma correlação forte entre estas variáveis. É possível que outros fatores expliquem melhor o aparecimento da dor 
lombopélvica na gestação. Atribui-se como uma de suas principais causas o hormônio relaxina, que resulta em articulações mais instáveis, predispondo as estruturas ao estresse e à dor ${ }^{40}$. É possível, também, que haja ativação constante dos músculos posteriores da coluna para compensar essa instabilidade, podendo levar à fadiga muscular e à dor lombar ${ }^{41}$. A dor lombopélvica também poderia ser causada por diminuição da resistência muscular dos músculos inferiores do tronco e extensores do quadril, que associada à ação da relaxina levaria a uma força de compressão ineficiente para estabilizar esta articulação ${ }^{42}$.

Em relação à qualidade de vida, a comparação do domínio físico do WHOQOL-bref entre o grupo controle e as gestantes no primeiro trimestre foi significativa. Além disso, pôde-se observar redução deste domínio do primeiro para o terceiro trimestre. Hueston e KasikMiller ${ }^{18}$ notaram que as pontuações das medidas físicas de saúde se alteraram durante a gestação.

É possível que logo no início da gestação a qualidade de vida das gestantes no aspecto físico tenha reduzido tanto pelas dores, como pelas alterações gastrointestinais ${ }^{43}$ e pela fadiga ${ }^{44}$. Do primeiro para o segundo trimestre, o escore do WHOQOL-bref praticamente se manteve. Isto pode ter ocorrido porque existem sintomas de vômitos, náuseas e fadiga ${ }^{44}$, além de sonolência diurna ${ }^{45}$. No terceiro trimestre o peso do feto e do líquido amniótico ${ }^{36}$, associado à fadiga ${ }^{44}$, à redução da mobilidade ${ }^{46} \mathrm{e}$ à sonolência ${ }^{45,47}$ podem ter levado à redução do domínio físico da qualidade de vida quando comparado ao primeiro trimestre.

\section{LIMITAÇÕES DO ESTUDO}

Com relação às limitações encontradas neste estudo, é importante relatar que apesar da fotogrametria ser um método de extrema importância por sua fácil aplicação clínica, baixo custo $^{48}$ e por ser válido para avaliar ângu$\operatorname{los}^{49}$, existe a possibilidade de variações em função da anatomia palpatória, traçado, forma de medição dos ângulos estudados ${ }^{48}$, postura assumida durante a tomada da foto ${ }^{50}$ e desvios do plano paralelo ${ }^{49}$.

\section{CONCLUSÃO}

Com o método de avaliação utilizado para analisar a postura das gestantes, não foi possível observar um padrão de alteração da curvatura lombar entre elas.
Também não foi encontrada relação entre alterações na curvatura lombar e a dor lombopélvica. Em relação à qualidade de vida, a comparação do domínio físico do WHOQOL-bref entre o grupo controle e as gestantes no primeiro trimestre foi significativa.

\section{REFERÊNCIAS}

1. Ritchie JR. Orthopedic considerations during pregnancy. Clin Obstet Gynecol. 2003:46(2):456-66.

2. Wu WH, Meijer OG, Uegaki K, Mens JM, van Dieën JH, Wuisman PI, et al. Pregnancy-related pelvic girdle pain (PPP), I: Terminology, clinical presentation, and prevalence. Eur Spine J. 2004:13(7):575-89.

3. Martins RF, Silva JLP. Prevalência de dores nas costas na gestação. Rev Assoc Med Bras. 2005;51(3):144-7.

4. Benetti FA, Pegoretti C, Wittig DS, Deprá PP, Campos MH, Sabatino $\mathrm{JH}$, et al. Curvatura lombar e inclinação do tronco durante o período gestacional. Rev Cienc Med. 2005;14(3):259-65.

5. Vleeming A, de Vries HJ, Mens JM, van Wingerden JP. Possible role of the long dorsal sacroiliac ligament in women with peripartum pelvic pain. Acta Obstet Gynecol Scand. 2002;81(5):430-6.

6. To WWK, Wong MWN. Factors associated with back pain symptoms in pregnancy and the persistence of pain 2 years after pregnancy. Acta Obstet Gynecol Scand. 2003;82(12):1086-91.

7. Ireland ML, Ott SM. The effects of pregnancy on the musculoskeletal system. Clin Orthop Relat Res. 2000;(372):169-79.

8. Rezende J. Obstetrícia. 3a ed. Rio de janeiro: Guanabara Koogan, 1992

9. Davis DC. The discomforts of pregnancy. J Obstet Gynecol Neonatal Nurs. 1996;25(1):73-81.

10. Nyska M, Sofer D, Porat A, Howard CB, Levi A, Meizner I. Planter foot pressures in pregnant women. Isr J Med Sci. 1997;33(2):139-46.

11. Munjin M, Llabaca F, Rojas J. Dolor lumbar relacionado al embarazo/ Pregnancy related low back pain. Ver Chil Obstet Ginecol. 2007;72(4):258-65.

12. Gazaneo MM, Oliveira LF. Alterações posturais durante a gestação/ Postural changes during pregnancy. Rev Bras Ativ Fís Saúde. 1998;3(2):13-21.

13. Ostgaard HC, Andersson GB, Schultz AB, Miller JA. Influence of some biomechanical factors on low back pain in pregnancy. Spine. 1993:18(1):61-5.

14. Kanayama N, el Maradny E, Kajiwara Y, Maehara K, Tokunaga N, Terao T. Hypolumbarlordosis: a predisposing factor for preeclampsia. Eur J Obstet Gynecol Reprod Biol. 1997;75(2):115-21.

15. Novaes FS, Shimo AKK, Lopes MHM. Lombalgia na gestação. Rev Lat Am Enfermagem. 2006;14(4):620-4.

16. Kristiansson P, Svärdsudd K, Schoultz BV. Back pain during pregnancy: a prospective study. Spine. 1996;21(6):702-9.

17. MacEvilly M, Buggy D. Back pain and pregnancy: a review. Pain. 1996;64(3):405-14

18. Hueston WJ, Kasik-Miller S. Changes in functional health status during normal pregnancy. J Fam Prac. 1998;47(3):209-12.

19. Olsson C, Nilsson-Wikmar L. Health-related quality of life and physical ability among pregnant women with and without back pain in late pregnancy. Acta Obstet Gynecol Scand. 2004;83(4):351-7. 
20. The WHOQOL Group. World Health Organization. WHOQOL: Measuring Quality of Life [dissertação]. Geneva: WHO; 1997.

21. Alexander GR, Tompkins ME, Cornely DA. Gestational age reporting and preterm delivery. Public Health Rep. 1990;105(3):267-75.

22. Rossavick LK, Fishburne Jl. Conceptional age, menstrual age, and ultrasound age: A second trimester comparison of pregnancies of known conceptional date with pregnancies dated from the last menstrual period. Obstet Gynecol. 1989;73(2):243-9.

23. Atalah SE, Castillo LC, Castro SR, Aldea AP. Propuesta de un nuevo estándar de evaluación nutricional en embarazadas. Rev Med Chil. 1997:125(12):1429-36.

24. Sato TO, Vieira ER, Coury HJCG. Análise da confiabilidade de técnicas fotométricas para medir a flexão anterior do tronco. Rev Bras Fisioter. 2003;7(1):53-59.

25. Hoppenfeld S. Propedêutica ortopédica: coluna e extremidades. São Paulo: Atheneu; 1998.

26. Pimenta CAM, Teixeira MJ. Questionário de dor McGill: proposta para adaptação para a língua portuguesa. Rev Bras Anestesiol. 1997:47(2):177-86.

27. Castro CES. A formulação linguística da dor: versão brasileira do questionário McGill de dor [dissertação]. São Carlos: Departamento de Fisioterapia, UFSCar; 1999

28. Fleck MPA, Louzada S, Xavier M, Chachamovich E, Vieira G, Santos L, et al. Desenvolvimento da versão em português do instrumento de avaliação de qualidade de vida da OMS (WHOQOL-100). Rev Bras Psiquiatr. 1999;2(1):19-28.

29. The WHOQOL Group. Development of the World Health Organization WHOQOL-bref. quality of life assessment. Psychol Med. 1998;28(3):551-8

30. Jacobson NS, Truax P. Clinical significance: A statistical approach to defining meaningful change in psychotherapy research. J Consult Clin Psychol. 1991;59(1):12-9.

31. Del Prette ZAP, Del Prette A. Significância clínica e mudança confiável: A efetividade das intervenções em psicologia. Psicol Teor Pesq. 2008:24(4):497-506.

32. Gilleard WL, Crosbie J, Smith R. Static trunk posture in sitting and standing during pregnancy and early postpartum. Arch Phys Med Rehabil. 2002:83(12):1739-44.

33. Moore K, Dumas GA, Reid JG. Postural changes associated with pregnancy and their relationship with low back pain. Clin Biomech. 1990;5(3):169-74.

34. Franklin ME, Conner-Kerr T. An Analysis of Posture and Back Pain in the First and Third Trimesters of Pregnancy. J Orthop Sports Phys Ther. 1998;28(3):133-8.

35. Bullock-Saxton JE. Changes in posture associated with pregnancy and the early post-natal period measured in standing. Physiother Theor Pract. 1991; 7(2):103-9.
36. Jensen RK, Doucet S, Treitz T. Changes in segment mass distribution during pregnancy. J Biomech. 1996;29(2):251-6.

37. Dumas GA, Reid JG, Wolfe LA, Griffin MP, McGrath MJ. Exercise, posture, and back pain during pregnancy. Clin Biomech. 1995;10(2):98-103.

38. Bullock JE, Jull GA, Bullock MI. The relationship of low back pain to postural changes during pregnancy. Aust J Physiother. 1987;33(1):10-77.

39. Garshasbi A, Zadeh SF. The effect of exercise on the intensity of low back pain in pregnant women. Int J Gynecol Obstet. 2005;88(3):271-5.

40. Ferreira CHJ, Nakano AMS. Reflexões sobre as bases conceituais que fundamentam a construção do conhecimento acerca da lombalgia na gestação. Rev Lat Am Enfermagem. 2001;9(3):95-100.

41. Sihvonen T, Huttunen M, Makkonen M, Airaksinen O. Functional changes in back muscles activity correlate with pain intensity and prediction of low back pain during pregnancy. Arch Phys Med Rehabil. 1998;79(10):1210-12.

42. Gutke A, Ostgaard HC, Oberg B. Association between muscle function and low back pain in relation to pregnancy. J Rehabil Med. 2008;40(4):304-11.

43. Broussard CN, Richter JE. Treating gastro-oesophageal reflux disease during pregnancy and lactation: what are the safest therapy options? Drug Saf. 1998;19(4):325-37.

44. Chou FH, Kuo SH, Wang RH. A longitudinal study of nausea and vomiting, fatigue and perceived stress in, and social support for, pregnant women through the three trimesters. Kaohsiung J Med Sci. 2008:24(6):306-14.

45. Schorr SJ, Chawla A, Devidas M, Sullivan CA, Naef RW 3rd, Morrison JC. Sleep patterns in pregnancy: a longitudinal study of polysomnography recordings during pregnancy. J Perinatol. 1998;18(6 Pt 1):427-30.

46. van de Pol G, de Leeuw JR, van Brummen HJ, Bruinse HW, Heintz AP, van der Vaart $\mathrm{CH}$. The Pregnancy Mobility Index: a mobility scale during and after pregnancy. Acta Obstet Gynecol Scand. 2006:85(7):786-91.

47. Borodulin K, Evenson KR, Monda K, Wen F, Herring AH, Dole N. Physical activity and sleep among pregnant women. Paediatr Perinat Epidemiol. 2010;24(1):45-52.

48. Souza FR, Ferreira F, Narciso FV, Makhoul CMB, Canto RST, Barauna MA. Avaliação da concavidade lombar pelo método radiográfico e pela cifolordometria. Rev Bras Fisioter. 2009;13(2): 103-9.

49. Sato TO, Moriguchi CS, Silva LCCB, Hansson GA, Coury HJCG. Validity and reliability of photogrammetry. In: XII Congresso Brasileiro de Biomecânica, 2007, São Pedro. Anais de Eventos do XII Congresso Brasileiro de Biomecânica, 2007.

50. Falcão FRC, Marinho APS, Sá KN. Correlação dos desvios posturais com dores músculoesqueléticas. Rev Cienc Med Biol. 2007;6(1):54-62. 